\title{
Association of Tumor Necrosis Factor Alpha, Interleukin 6, and C- Reactive Protein with the Risk of Developing Type 2 Diabetes: A Retrospective Cohort Study of Rural Thais
}

\author{
Jirayu Lainampetch, ${ }^{1}$ Pornpimol Panprathip, ${ }^{1}$ Chanchira Phosat, ${ }^{2}$ \\ Noppanath Chumpathat, ${ }^{3}$ Pattaneeya Prangthip, ${ }^{1}$ Ngamphol Soonthornworasiri, ${ }^{4}$ \\ Somchai Puduang, ${ }^{1}$ Naruemon Wechjakwen, ${ }^{5}$ and Karunee Kwanbunjan ${ }^{1}{ }^{1}$ \\ ${ }^{1}$ Department of Tropical Nutrition and Food Science, Faculty of Tropical Medicine, Mahidol University, Bangkok 10400, Thailand \\ ${ }^{2}$ Department of Nutrition, Faculty of Public Health, Mahidol University, Bangkok 10400, Thailand \\ ${ }^{3}$ Faculty of Nursing, Huachiew Chalermprakiet University, Samut Prakan 10540, Thailand \\ ${ }^{4}$ Department of Tropical Hygiene, Faculty of Tropical Medicine, Mahidol University, Bangkok 10400, Thailand \\ ${ }^{5}$ Faculty of Public Health, Nakhon Ratchasima Rajabhat University, Nakhon Ratchasima 30000, Thailand
}

Correspondence should be addressed to Karunee Kwanbunjan; karunee.kwa@mahidol.ac.th

Received 19 April 2019; Accepted 30 July 2019; Published 8 August 2019

Academic Editor: Paolo Fiorina

Copyright (c) 2019 Jirayu Lainampetch et al. This is an open access article distributed under the Creative Commons Attribution License, which permits unrestricted use, distribution, and reproduction in any medium, provided the original work is properly cited.

\begin{abstract}
The linkage of obesity, inflammation, and type 2 diabetes mellitus (T2DM) has been extensively investigated for over a decade. However, the association between inflammatory biomarkers, including C-reactive protein (CRP), interleukin 6 (IL-6), and tumor necrosis factor alpha (TNF- $\alpha$ ), and T2DM is still inconsistent and limited. Thus, this study is aimed at elucidating the association between inflammatory marker levels and the risk of developing T2DM in many aspects. Among 296 subjects enrolled in 2013, 248 non-T2DM subjects who were completely reinvestigated in 2014 and 2015 were included in a 2-year retrospective analysis. Multivariate logistic regression was performed to evaluate the association of baseline inflammatory marker levels and variation with incidence of T2DM. After the 2-year follow-up, 18.6\% of total subjects had developed T2DM. The risk of developing T2DM was significantly increased in subjects with a high level of baseline CRP (OR $=4.02,95 \%$ CI: 1.77 9.12, $P=0.001)$, and a stronger impact was found with the combination of high CRP and IL-6 levels $(\mathrm{OR}=5.11,95 \% \mathrm{CI}: 1.27$ 20.49, $P=0.021)$. One-year inflammatory marker variation analysis also revealed the significant association of elevated TNF- $\alpha$ and risk of developing T2DM ( $\mathrm{OR}=4.88,95 \% \mathrm{CI}$ : 1.01-23.49, $P=0.048)$. In conclusion, besides consideration of CRP levels alone, our findings suggested that IL-6 outstandingly plays a contributing role in T2DM progression and elevated TNF- $\alpha$ levels over time could be a potential predictor of T2DM.
\end{abstract}

\section{Introduction}

Diabetes is an important cause of morbidity and mortality that leads the top ten public health burdens in Thailand [1]. According to the recent report in 2017, the prevalence of diabetes was $8.3 \%$ among a nationwide adult population, representing over 4 million diabetic cases in Thailand [2]. The northeastern region was estimated to have the highest morbidity rate, especially in those aged $35-60$ years [3]. This may be due to the widespread transition from an agricultural to an industrial society in this region that changes lifestyle habits and subsequently contributes to metabolic disease.

C-reactive protein (CRP), a major acute-phase protein, is generally considered as an indicator for low-grade systemic inflammation in chronic diseases such as cancer and cardiovascular disease (CVD) [4]. In regard to T2DM, CRP is also known as an independent predictor of T2DM [5-9]. Previous studies have extensively reported the association of elevated CRP levels with insulin resistance and progression of T2DM [6, 10-12]. 
High caloric intake with physical inactivity causes excess fat accumulation, indicating as the major risk factor of insulin resistance and type 2 diabetes mellitus (T2DM) [13]. Evidences showed that hypertrophied adipocytes involve with an inflammatory condition [14-18]. Inflammatory cytokines secreted by adipose tissue, such as interleukin 6 (IL-6) and tumor necrosis factor alpha (TNF- $\alpha$ ), may exert an endocrine effect to promote insulin resistance by interfering with the insulin signalling pathway, leading to the clinical manifestation of T2DM $[19,20]$.

Clinical findings revealed the role of IL- 6 and TNF- $\alpha$ in glucose homeostasis and metabolism and also the indirectly possible action on the pancreatic $\beta$-cell $[21,22]$. Furthermore, the presence of these cytokines leads to inflammatory responses with elevated CRP [23-25]. IL-6 and TNF- $\alpha$ were suggested as a possible marker for T2DM prediction [26-28]. However, nonstatistically significant results have also been reported $[8,12,29]$. The biological role of these cytokines on T2DM development may still be uncertain.

The linkage between inflammation and T2DM which was found in a cross-sectional study showed difficulty in considering the sequence of events and may not reflect the true risk of disease. A single baseline measurement of CRP, IL-6, and TNF- $\alpha$ levels was unable to evaluate the effects of changes over time, and no evidence of interaction between these inflammatory markers was found. We therefore designed a cohort study with different analyses to determine the association between inflammatory marker levels and the risk of developing T2DM among Thai adults in the rural area of the northeastern region where urbanization and industrialization are rapidly growing. Our study was aimed at evaluating the potential of the T2DM predictor in longitudinal analysis.

\section{Materials and Methods}

2.1. Study Design and Data Source. A retrospective cohort study on rural Thai was carried out in 12 villages of Sung Noen District, Nakhon Ratchasima Province, from 2013 to 2015. The data set was extracted from 296 participants, consisting of demographic information, dietary intake, anthropometric assessments, and biochemical analysis. The description of subject characteristics, selection criteria, and data collection have been published elsewhere [12].

2.2. Study Subjects. This study includes nondiabetic subjects aged 35-66 years who were initially examined in 2013 (baseline) and subsequently reexamined based on identical procedures in 2014 and 2015. After excluding those who identified as T2DM at baseline by fasting blood glucose (FBG), 2-hour blood glucose (2-h BG), and glycated hemoglobin (HbAlc) [30], 248 eligible subjects who completed on reexamination in 2014 and 2015 were entered for longitudinal analysis. The study protocol was approved by the Ethics Committee of the Faculty of Tropical Medicine, Mahidol University (TMEC 18-040).

2.3. Criteria of Main Outcome: Incidence of T2DM. Incident cases of T2DM were determined as FBG $\geq 126 \mathrm{mg} / \mathrm{dl}$
(7.0 mmol/l), $2-\mathrm{h} \mathrm{BG} \geq 200 \mathrm{mg} / \mathrm{dl}(11.1 \mathrm{mmol} / \mathrm{l})$, and $\mathrm{HbA}$ $1 c \geq 6.5 \%(48 \mathrm{mmol} / \mathrm{mol})$ at reexamination according to the American Diabetes Association (ADA) criteria [30] or with a clinical diagnosis for T2DM during the follow-up period. A total of 30 new cases of T2DM were found in the 2014 reexamination; 16 new cases were found in the 2015 reexamination.

2.4. Statistical Analysis. Continuous variables were expressed as median (interquartile range) and the differences were compared by Mann-Whitney $U$ test. For categorical variables, data were reported as proportions (\%) and compared using chi-squared tests. To investigate the relationship of inflammation and T2DM, univariate and multivariate logistic regressions were used to calculate the odds ratios (ORs) and $95 \%$ confidence intervals (CIs) of the baseline inflammatory marker levels for 1-year and 2-year progressions of T2DM. The role of inflammatory marker interaction was examined. Baseline TNF- $\alpha$ and IL- 6 levels were classified using the tertile as the cut-off point (setting the subjects who were $1^{\text {st }}$ tertile for both TNF- $\alpha$ and IL- 6 as the low level and those who were $3^{\text {rd }}$ tertile as the high level), and baseline CRP levels were classified by suggested cut-off values [31]. ORs and 95\% CIs were estimated for each combination, including two and three of these inflammatory markers. In addition, the association between changes in inflammatory marker levels from baseline (2013) to 2014 and the risk of developing T2DM at 2 years was analyzed. Changes in serum inflammatory marker levels were divided into four categories: low level in both 2013 and 2014, low level in 2013 but high in 2014, high level in 2013 but low in 2014, and high level in both 2013 and 2014. All statistical analyses were performed using SPSS (version 18.0; SPSS, Chicago, IL, USA). A $P$ value $<0.05$ was considered statistically significant.

\section{Results}

3.1. Baseline Characteristics of Study Subjects. A total of 248 diabetes-free individuals composed of 82 males and 166 females with fully completed data collections and laboratory analyses in 2013 and who were completely reexamined in 2014 and 2015 were included. They were divided into two groups according to incidence of T2DM as follows: the nonprogression group: subjects who were diabetes-free throughout the study period, and the progression group: subjects who were identified as T2DM at any reexamination year. Table 1 displays baseline demographic and anthropometric, biochemical, clinical, and dietary characteristics of the subjects with or without T2DM progression at reexamination. Age of new-onset T2DM participants was rather older $(P=0.035)$, and half of them had a diabetes family history. Blood glucose and insulin resistance results at baseline in the T2DM groups substantially showed greater values than the normal group. Furthermore, their baseline BMI, WC, WHR, VF, DBP, and triglyceride were elevated higher but with lower baseline HDL-C levels. However, dietary intake assessed from a semifood frequency questionnaire (semiFFQ) showed no statistical difference between the two groups. In regard to inflammatory markers, subjects with 
TABLE 1: Baseline characteristics of study subjects in longitudinal analysis based on progression to T2DM.

\begin{tabular}{|c|c|c|c|}
\hline & Nonprogression group & Progression group & $P$ value \\
\hline Number & 202 & 46 & \\
\hline Age (years) & $45(40-49)$ & $47.5(42-52)$ & 0.035 \\
\hline Gender (male/female) & $66 / 136$ & $16 / 30$ & 0.784 \\
\hline Current smoker (\%) & $40(19.8 \%)$ & $10(21.7 \%)$ & 0.956 \\
\hline Alcoholic drinking (\%) & $92(45.5 \%)$ & $18(39.1 \%)$ & 0.498 \\
\hline Lack of exercise (\%) & $54(26.7 \%)$ & $12(26.1 \%)$ & 0.614 \\
\hline Family history of diabetes (\%) & $71(35.1 \%)$ & $23(50.0 \%)$ & 0.061 \\
\hline BMI $\left(\mathrm{kg} / \mathrm{m}^{2}\right)$ & $25.17(22.11-27.85)$ & $27.08(22.37-31.11)$ & 0.020 \\
\hline $\mathrm{WC}(\mathrm{cm})$ & $83.00(75.00-89.00)$ & $89.25(77.00-95.50)$ & 0.003 \\
\hline WHR & $0.87(0.83-0.92)$ & $0.90(0.85-0.95)$ & 0.014 \\
\hline Body fat (\%) & $30.65(23.75-35.10)$ & $33.00(25.53-37.63)$ & 0.058 \\
\hline Visceral fat (\%) & $8.50(6.00-12.50)$ & $11.00(7.88-16.88)$ & 0.008 \\
\hline Muscle (\%) & $25.45(23.60,30.40)$ & $24.75(22.60-29.50)$ & 0.078 \\
\hline $\mathrm{SBP}(\mathrm{mmHg})$ & $120(111-129)$ & $123(114-130)$ & 0.184 \\
\hline $\mathrm{DBP}(\mathrm{mmHg})$ & $76(70-81)$ & $78(73-86)$ & 0.046 \\
\hline FBG (mg/dl) & $90(87-98)$ & $103(93-117)$ & $<0.001$ \\
\hline 2-h BG (mg/dl) & $113(98-140)$ & $173(117-185)$ & $<0.001$ \\
\hline $\mathrm{HbA1C}(\%)$ & $5.3(4.9-5.6)$ & $5.6(5.2-6.1)$ & $<0.001$ \\
\hline HOMA-IR & $1.18(0.80-1.59)$ & $1.63(1.10-2.68)$ & 0.001 \\
\hline HOMA- $\beta$ & $66.17(43.64-97.75)$ & $56.66(26.33-106.70)$ & 0.158 \\
\hline Triglyceride (mg/dl) & $120(89-170)$ & $139(111-227)$ & 0.017 \\
\hline Total cholesterol (mg/dl) & $200(173-222)$ & $203(178-225)$ & 0.822 \\
\hline HDL-C (mg/dl) & $52(42-62)$ & $45(36-54)$ & 0.005 \\
\hline LDL-C (mg/dl) & $122(96-149)$ & $120(93-143)$ & 0.705 \\
\hline CRP (mg/dl) & $1.39(0.61-3.03)$ & $2.81(1.48-6.65)$ & 0.001 \\
\hline IL-6 (pg/ml) & $14.51(4.31-248.93)$ & $18.53(5.25-732.45)$ & 0.268 \\
\hline TNF- $\alpha(\mathrm{pg} / \mathrm{ml})$ & $53.80(27.22-79.72)$ & $62.28(31.28-89.87)$ & 0.272 \\
\hline Daily caloric intake (kcal/day) & $2181.20(1516.80-2900.81)$ & $2071.50(1581.53-3118.68)$ & 0.910 \\
\hline Carbohydrate intake (\% of DCI) & $66.89(60.30-72.18)$ & $67.49(61.10-73.95)$ & 0.779 \\
\hline Protein intake (\% of DCI) & $11.03(9.78-12.53)$ & $10.90(9.87-12.57)$ & 0.906 \\
\hline Fat intake (\% of DCI) & $20.38(15.27-25.90)$ & $19.97(15.00-24.83)$ & 0.645 \\
\hline Total dietary GL (per day) & $186.80(125.80-279.97)$ & $207.44(132.48-248.02)$ & 0.778 \\
\hline
\end{tabular}

Data presented as median (interquartile range) or number (\%). Abbreviations: BMI: body mass index; WC: waist circumference; WHR: waist-hip ratio; SBP: systolic blood pressure; DBP: diastolic blood pressure; FBG: fasting blood glucose; 2-h BG: 2-hour blood glucose after $75 \mathrm{~g}$ oral glucose tolerance test; HbAlc: hemoglobin A1c; HOMA-IR: homeostatic model assessment of insulin resistance; HOMA- $\beta$ : homeostatic model assessment of $\beta$-cell function; HDL-C: high-density lipoprotein cholesterol; LDL-C: low-density lipoprotein cholesterol; CRP: C-reactive protein; IL-6: interleukin 6; TNF- $\alpha$ : tumor necrosis factor alpha; DCI: daily caloric intake; GL: glycemic load.

T2DM obviously indicated a higher baseline CRP level, but the difference of IL-6 and TNF- $\alpha$ levels between groups was not statistically significant.

3.2. Relationship of Anthropometric and Metabolic Parameters with Serum Inflammatory Markers at Baseline. Using the Mantel-Haenszel chi-squared trend test, we found that low physical activity subjects (exercise less than 3 times per week) indicated higher proportions of highest IL- 6 and TNF- $\alpha$ levels than the physically active group (40.2\% vs. $28.4 \%, P$ for trend $=0.014$, and $43.3 \%$ vs. $26.5 \%, P$ for trend $=0.006$, respectively). Likewise, low physical activity subjects showed a higher CRP level (68.9\% vs. $43.3 \%)$ compared with individuals who exercise frequently $(P<0.001)$. Further- more, Spearman's rank correlation coefficients were calculated. Serum CRP correlated significantly with WHR $(r=0.213, P=0.001)$, whereas IL- 6 and TNF- $\alpha$ only showed a positive trend towards correlation with WHR $(r=0.094$ and $r=0.123$, respectively). CRP showed a positive correlation with HOMA-IR $(r=0.322, \quad P<0.001)$ and FBG $(r=0.131, P=0.040)$, and IL-6 positively correlated with FBG $(r=0.126, P=0.047)$. Significant correlations were also considerably found between CRP and other parameters, including BMI $(r=0.325)$, WC $(r=0.359)$, total cholesterol $(r=0.132)$, HDL-C $(r=-0.177)$, and LDL-C $(r=0.169)$.

3.3. Predictive Effect of Baseline Serum Inflammatory Markers on Blood Glucose Status. A linear regression model was 
TABLE 2: Multiple regression analysis of blood glucose indices vs. 5 independent variables among 248 subjects at baseline.

\begin{tabular}{|c|c|c|c|c|c|c|}
\hline \multirow{2}{*}{ Variables } & \multicolumn{2}{|l|}{ FBG $(\mathrm{mg} / \mathrm{dl})^{*}$} & \multicolumn{2}{|l|}{ 2-h BG (mg/dl)** } & \multicolumn{2}{|l|}{$\operatorname{HbA1C}(\%)^{* * *}$} \\
\hline & Standardized coefficients $(\beta)$ & $P$ value & Standardized coefficients $(\beta)$ & $P$ value & Standardized coefficients & $P$ value \\
\hline Age (years) & 0.163 & 0.010 & 0.120 & 0.057 & 0.188 & 0.003 \\
\hline Sex & -0.129 & 0.044 & 0.155 & 0.015 & 0.079 & 0.215 \\
\hline $\mathrm{CRP}(\mathrm{mg} / \mathrm{dl})$ & 0.138 & 0.028 & 0.216 & 0.001 & 0.223 & $<0.001$ \\
\hline IL-6 (pg/ml) & 0.137 & 0.043 & 0.059 & 0.376 & -0.054 & 0.420 \\
\hline TNF- $\alpha(\mathrm{pg} / \mathrm{ml})$ & 0.010 & 0.878 & 0.041 & 0.542 & 0.084 & 0.216 \\
\hline
\end{tabular}

Abbreviations: FBG: fasting blood glucose; 2-h BG: 2-hour blood glucose after $75 \mathrm{~g}$ oral glucose tolerance test; HbAlc: hemoglobin Alc; CRP: C-reactive protein; IL-6: interleukin 6; TNF- $\alpha$ : tumor necrosis factor alpha. ${ }^{*} F=4.759$ and $P<0.001$ vs. 5 independents. ${ }^{* *} F=4.800$ and $P<0.001$ vs. 5 independents. ${ }^{* * *} F=5.037$ and $P<0.001$ vs. 5 independents.

TABLE 3: Odds ratios (ORs) and 95\% confidence intervals (CIs) for 2-year progression of type 2 diabetes according to baseline serum inflammatory marker levels.

\begin{tabular}{|c|c|c|c|c|}
\hline \multirow{2}{*}{ Variables } & \multicolumn{2}{|c|}{ Univariate analysis } & \multicolumn{2}{|c|}{ Multivariate analysis } \\
\hline & Crude OR (95\% CI) & $P$ value & Adjusted OR* (95\% CI) & $P$ value \\
\hline \multicolumn{5}{|l|}{ CRP (mg/dl) } \\
\hline Low level $(<1.00)$ & 1 & & 1 & \\
\hline High level $(\geq 1.00)$ & $4.20(1.92-9.14)$ & $<0.001$ & $4.02(1.77-9.12)$ & 0.001 \\
\hline \multicolumn{5}{|l|}{ IL-6 (pg/ml) } \\
\hline $1^{\text {st }}$ tertile $(<5.93)$ & 1 & & 1 & \\
\hline $2^{\text {nd }}$ tertile $(5.93-85.65)$ & $1.19(0.53-2.68)$ & 0.679 & $1.08(0.46-2.52)$ & 0.828 \\
\hline $3^{\text {rd }}$ tertile $(>85.65)$ & $1.51(0.69-3.34)$ & 0.303 & $1.57(0.68-3.59)$ & 0.291 \\
\hline \multicolumn{5}{|l|}{$\mathrm{TNF}-\alpha(\mathrm{pg} / \mathrm{ml})$} \\
\hline $1^{\text {st }}$ tertile $(<36.47)$ & 1 & & 1 & \\
\hline $2^{\text {nd }}$ tertile $(36.47-73.30)$ & $1.41(0.62-3.21)$ & 0.408 & $1.35(0.57-3.19)$ & 0.489 \\
\hline $3^{\text {rd }}$ tertile $(>73.30)$ & $1.66(0.74-3.72)$ & 0.215 & $1.63(0.70-3.79)$ & 0.260 \\
\hline
\end{tabular}

performed to elucidate the metabolic correlation. In a multivariable regression analysis, with FBG, 2-h BG, and $\mathrm{HbA1C}$ as dependent variables and age, sex, CRP, IL-6, and TNF- $\alpha$ as independent variables, significant associations were shown between FBG and age, sex, CRP, and IL-6 $(F=4.759, P<0.001)$ but not TNF- $\alpha$ level. For $2-\mathrm{h}$ BG and $\mathrm{HbA} 1 \mathrm{C}, \mathrm{CRP}$ showed the strong associations with these indices, implying as a good predictor for blood glucose status specified by FBG, $2-\mathrm{h} \mathrm{BG}$, and $\operatorname{HbA1C}(\beta=0.216, P=0.001$, and $\beta=0.223, P<0.001$, respectively) (Table 2 ).

3.4. The Association between Baseline Serum Inflammatory Marker Levels and the Risk of Developing T2DM. The results of logistic regression for the 2-year T2DM progression are presented in Table 3. Study subjects with CRPlevels $\geq 1$ $\mathrm{mg} / \mathrm{dl}$ had approximately a fourfold $(\mathrm{OR}=4.20,95 \% \mathrm{CI}$ : 1.92-9.14, $P<0.001$ ) greater risk of developing T2DM when compared to those in the lower level. Highest IL-6 and TNF- $\alpha$ level groups were more likely to increase the risk of developing T2DM ( $\mathrm{OR}=1.66,95 \% \mathrm{CI}$ : $0.74-3.72$, and $\mathrm{OR}=1.51,95 \% \mathrm{CI}$ : 0.69-3.34, respectively). After adjusting for confounders in the multivariate analysis, a statistically increased risk of developing T2DM was obviously found in subjects with elevated CRP levels (OR $=4.02$, 95\% CI: $1.77-9.12, P=0.001)$. Although there was no statistically significant association, a trend towards elevated risk of developing T2DM was observed in subjects with the highest TNF- $\alpha$ and IL- 6 concentration ranges.

3.5. Combination Effects of Baseline Serum Inflammatory Marker Levels on the Risk of Developing T2DM. Since consideration of a single inflammatory marker alone may not show the statistical relationship with risk of developing T2DM, we therefore performed the combination analysis to evaluate the interaction effect in each inflammatory marker. The results of logistic regression for the 2-year T2DM progression are presented in Table 4. The association was obviously found in the synergistic interaction term between the high level of baseline CRP and IL-6; subjects with this combination had a substantially increased risk of T2DM by almost 6 times compared with the low-level group $(\mathrm{OR}=5.96,95 \% \mathrm{CI}$ : 1.55-22.88, $P=0.009)$. The effects were still statistically significant after adjusting for confounders; the synergistic interaction value was $5.1(\mathrm{OR}=5.11,95 \% \mathrm{CI}: 1.27-20.49$, $P=0.021)$. Subjects with a combined elevation of baseline $\mathrm{CRP}$ and TNF- $\alpha$ levels indicated a significantly increased risk of developing T2DM $(\mathrm{OR}=4.11,95 \% \mathrm{CI}$ : $1.10-15.33$, $P=0.035)$ when compared with the low-level group in the adjusted analysis. Conversely, the combination of elevated levels of baseline IL- 6 and TNF- $\alpha$ was not significantly 
TABLE 4: Odds ratios (ORs) and 95\% confidence intervals (CIs) for 2-year progression of type 2 diabetes according to interaction between baseline serum CRP, IL- 6 , and TNF- $\alpha$ levels.

\begin{tabular}{|c|c|c|c|c|}
\hline \multirow{2}{*}{ Variables } & \multicolumn{2}{|c|}{ Univariate analysis } & \multicolumn{2}{|c|}{ Multivariate analysis } \\
\hline & Crude OR $(95 \%$ CI $)$ & $P$ value & Adjusted OR* (95\% CI) & $P$ value \\
\hline \multicolumn{5}{|l|}{ Interaction of $\mathrm{CRP}^{\mathrm{a}}$ and IL- ${ }^{\mathrm{b}}$} \\
\hline CRP (low)+IL-6 (low) & 1 & & 1 & \\
\hline CRP (low)+IL-6 (high) & $2.28(0.62-8.45)$ & 0.218 & $1.78(0.45-7.00)$ & 0.412 \\
\hline CRP (high)+IL-6 (low) & $2.65(0.67-10.47)$ & 0.164 & $2.01(0.48-8.49)$ & 0.340 \\
\hline CRP (high)+IL-6 (high) & $5.96(1.55-22.88)$ & 0.009 & $5.11(1.27-20.49)$ & 0.021 \\
\hline \multicolumn{5}{|l|}{ Interaction of CRP and TNF- $\alpha^{\mathrm{c}}$} \\
\hline CRP (low) + TNF- $\alpha$ (low) & 1 & & 1 & \\
\hline CRP (low)+TNF- $\alpha$ (high) & $1.61(0.49-5.26)$ & 0.429 & $1.51(0.44-5.16)$ & 0.509 \\
\hline CRP (high)+TNF- $\alpha$ (low) & $1.23(0.34-4.47)$ & 0.756 & $1.17(0.31-4.46)$ & 0.822 \\
\hline CRP (high)+TNF- $\alpha$ (high) & $4.05(1.18-13.85)$ & 0.026 & $4.11(1.10-15.33)$ & 0.035 \\
\hline \multicolumn{5}{|l|}{ Interaction of IL- 6 and TNF- $\alpha$} \\
\hline IL-6 (low)+TNF- $\alpha$ (low) & 1 & & 1 & \\
\hline IL-6 (low)+TNF- $\alpha$ (high) & $1.37(0.55-3.40)$ & 0.504 & $1.28(0.50-3.32)$ & 0.607 \\
\hline IL-6 (high)+TNF- $\alpha$ (low) & $2.05(0.44-9.47)$ & 0.359 & $1.65(0.33-8.42)$ & 0.544 \\
\hline IL-6 (high)+TNF- $\alpha$ (high) & $1.99(0.71-5.58)$ & 0.190 & $2.34(0.79-6.97)$ & 0.127 \\
\hline \multicolumn{5}{|l|}{ Interaction of CRP, IL- 6 and TNF- $\alpha$} \\
\hline CRP (low)+IL-6 (low)+TNF- $\alpha$ (low) & 1 & & 1 & \\
\hline CRP (high)+IL-6 (high)+TNF- $\alpha$ (high) & $5.71(1.07-30.63)$ & 0.042 & $4.77(0.84-27.11)$ & 0.078 \\
\hline
\end{tabular}

Abbreviations: CRP: C-reactive protein; IL-6: interleukin 6; TNF- $\alpha$ : tumor necrosis factor alpha. ${ }^{*}$ OR was adjusted for age, gender, WHR, VF, and TG. ${ }^{2} \mathrm{CRP}$ (low): serum CRP $<1.00 \mathrm{mg} / \mathrm{dl}$; CRP (high): serum CRP $\geq 1.00 \mathrm{mg} / \mathrm{dl}$. ${ }^{\text {b }} \mathrm{IL}-6$ (low): serum IL-6 in $1^{\text {st }}$ tertile ( $\left.<5.93 \mathrm{pg} / \mathrm{ml}\right) ;$ IL-6 (high): serum IL-6 in $3^{\text {rd }}$ tertile ( $>85.65 \mathrm{pg} / \mathrm{ml}) .{ }^{\mathrm{c}}$ TNF- $\alpha$ (low): serum TNF- $\alpha$ in $1^{\text {st }}$ tertile $(<36.47 \mathrm{pg} / \mathrm{ml})$; TNF- $\alpha$ (high): serum TNF- $\alpha$ in $3^{\text {rd }}$ tertile $(>73.30 \mathrm{pg} / \mathrm{ml})$.

associated with T2DM; however, subjects in this group showed a slight trend towards increased risk of developing T2DM (OR = 2.34, 95\% CI: 0.79-6.97, $P=0.127)$. Furthermore, we performed an analysis of synergistic interaction among all 3 inflammatory markers. The effect of combined high CRP, IL-6, and TNF- $\alpha$ levels was found with a significantly increased risk to develop T2DM in the crude analysis $(\mathrm{OR}=5.71,95 \% \mathrm{CI}$ : $1.07-30.63, P=0.042)$, whereas this relationship was not rather strong after the adjustment of confounding factors.

3.6. The Association between Changes in Serum Inflammatory Marker Levels and the Risk of Developing T2DM. As displayed in Table 5, subjects with a consistently high level of $\mathrm{CRP}$ in 1 year had approximately 4 times $(\mathrm{OR}=4.23,95 \%$ CI: $1.54-11.59$, and $P=0.005)$ greater risk of developing T2DM, compared to those with a consistently low level. After adjusting for confounders, high CRP levels remained a significant association with increased risk of developing T2DM (OR $=4.22$, 95\% CI: $1.45-12.31, P=0.008)$. T2DM risk significantly increased in subjects with high IL-6 levels at both baseline and the first year of reexamination $(\mathrm{OR}=4.14,95 \% \mathrm{CI}:$ 1.06-16.21), and elevated IL-6 levels for 1 year showed a trend towards increased risk of developing T2DM. However, these trends attenuated after the adjustment of confounding factors. Considering changes in TNF- $\alpha$ levels, the risk of developing T2DM after 2 years significantly increased by about 5 times in subjects with high TNF- $\alpha$ levels at the first year of reexamination, no matter if the levels were low or high at baseline. Moreover, a statistical significant effect was also found after adjusting for confounders $(\mathrm{OR}=4.88,95 \% \mathrm{CI}$ : $1.01-23.49, P=0.048$ for low to high, and OR $=3.58,95 \% \mathrm{CI}$ : $1.02-12.55, P=0.047$ for high to high).

\section{Discussion}

In this retrospective cohort study, we primarily found the positive predictive power of inflammatory markers on blood glucose concentrations. The results further showed the evidence that the risk of developing T2DM was significantly increased among subjects with elevated baseline CRP levels, and this association was stronger in combination between high levels of baseline CRP and IL-6. Additionally, we also pointed that a notable association between one-year elevations of TNF- $\alpha$ levels and T2DM progression among the participants represents the Thai population in a rural area where nutrition transition occurs.

As increasing age, overweight, impaired glucose tolerance, and family history have been suggested to be major risk determinants for T2DM [2], older subjects in this study were more likely to develop T2DM than the younger group. Statistical outcome displayed baseline BMI, WC, WHR, and blood glucose values higher in the participants with T2DM progression. Although it did not reach statistical significance, the T2DM group indicated a trend towards more family members suffering from diabetes. These diabetes risk factors should be firstly emphasized for planning a disease prevention program in this cohort. In contrast, some well-known modifiable factors, including smoking, physical inactivity, 
TABLE 5: Odds ratios (ORs) and 95\% confidence intervals (CIs) for 2-year progression of type 2 diabetes according to 1-year changes of serum inflammatory marker levels.

\begin{tabular}{|c|c|c|c|c|}
\hline \multirow{2}{*}{ Variables } & \multicolumn{2}{|c|}{ Univariate analysis } & \multicolumn{2}{|c|}{ Multivariate analysis } \\
\hline & Crude OR (95\% CI) & $P$ value & Adjusted OR* (95\% CI) & $P$ value \\
\hline \multicolumn{5}{|l|}{ 1-year changes of serum $\mathrm{CRP}^{\mathrm{a}}$} \\
\hline Low (baseline)-low (1 year) & 1 & & 1 & \\
\hline Low (baseline)-high (1 year) & $0.66(0.17-2.59)$ & 0.548 & $0.65(0.16-2.60)$ & 0.539 \\
\hline High (baseline)-high (1 year) & $4.23(1.54-11.59)$ & 0.005 & $4.22(1.45-12.31)$ & 0.008 \\
\hline \multicolumn{5}{|l|}{1 year changes of serum IL- $6^{\mathrm{b}}$} \\
\hline Low (baseline)-low (1 year) & 1 & & 1 & \\
\hline Low (baseline)-high (1 year) & $4.17(0.88-19.82)$ & 0.073 & $3.36(0.67-16.94)$ & 0.142 \\
\hline High (baseline)-high (1 year) & $4.14(1.06-16.21)$ & 0.041 & $3.68(0.91-14.90)$ & 0.068 \\
\hline \multicolumn{5}{|l|}{ 1-year changes of serum TNF- $\alpha^{c}$} \\
\hline Low (baseline)-low (1 year) & 1 & & 1 & \\
\hline Low (baseline)-high (1 year) & $5.28(1.18-23.71)$ & 0.030 & $4.88(1.01-23.49)$ & 0.048 \\
\hline High (baseline)-high (1 year) & $4.12(1.22-13.93)$ & 0.023 & $3.58(1.02-12.55)$ & 0.047 \\
\hline \multicolumn{5}{|c|}{ 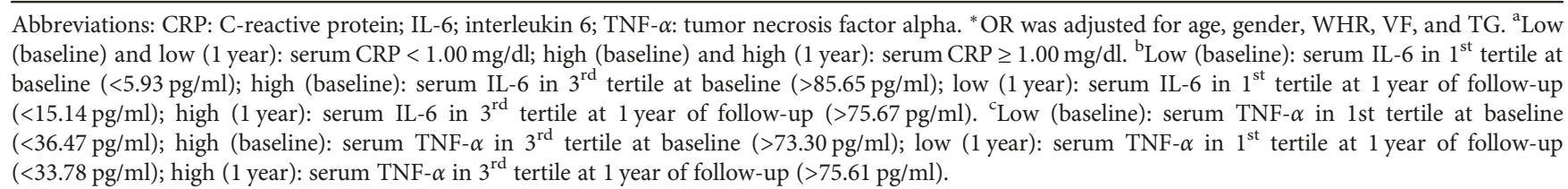 } \\
\hline
\end{tabular}

and unhealthy food intake, were not found to have any association with the development of T2DM in the present study. These nonstatistically significant results were probably due to self-reported data depending on participants' memories.

Focusing on inflammatory markers, the difference of baseline CRP, IL-6, and TNF- $\alpha$ levels between the normal and T2DM progression groups was found in our study. This is consistent with previous findings which showed the positive correlation of inflammatory markers and blood glucose indices $[10,12,29]$. Moreover, a predictive analysis using multiple linear regression significantly revealed that CRP and IL-6 levels at baseline could be possible biomarkers for predicting blood glucose. This may imply their certain role in relation to the hyperglycemic state. Primary outcomes of baseline parameters in the present study provided a basic assumption regarding directionality in the association of inflammatory marker levels with glucose homeostasis.

As elevated CRP levels were suggested as an independent predictor for incident T2DM [5-9], a recent study conducted in the same cohort also showed the significant association of CRP levels and T2DM [12]; however, this cross-sectional analysis may not clearly describe that CRP is the actual supportive cause for the development of T2DM. In the present study, a four times higher risk of developing T2DM by baseline CRP could establish a temporal relationship, the time sequence between a factor and disease, among this cohort. As with other longitudinal analyses suggesting the effect of stable CRP levels during a long-term period [32, 33], our study also found the corresponding result which was constantly elevated for 1 year. These findings support the hypothesis that CRP may have indirect effect on insulin sensitivity and insulin production from pancreatic $\beta$-cells through the alteration of innate immune response due to elevated systemic inflammation [34]. High levels of CRP are also involved in the production of adhesion molecules, namely, E-selectin, ICAM-1, and VCAM-1, which play a role in vascular endothelial dysfunction, insulin transport reduction, and peripheral insulin resistance [35]. The positive relationship of CRP and T2DM progression probably relates to atherosclerosis development. However, our study completely excluded subjects with CVD at baseline examination, and new-onset CVD was not reported during the follow-up period.

Chronic low-grade inflammatory processes are initiated by the accumulated fat in adipocytes. While the activation of inflammation is constantly increasing, inflammatory cells, mainly macrophages, are recruited to infiltrate in adipose tissue. Macrophages take responsibility for producing and secreting inflammatory cytokines such as IL-6 and TNF- $\alpha$ $[15,16,20]$. Elevations in these cytokine levels have direct influence on insulin signal intensity, contributing to insulin resistance in the target cell. Impaired insulin signal transduction may be explained by a principal molecular mechanism, the serine phosphorylation of insulin receptor substrate 1 (IRS-1), which mediates insulin resistance through inhibitory effects on glucose transporter 4 (GLUT4) [36-38]. Indirect effects of IL- 6 and TNF- $\alpha$ are also found by inducing CRP production through upregulation of transcription factors [39]. Thus, these inflammatory cytokines are involved in obesity-related T2DM. However, this study, as well as a previous study with the same cohort [12], reported weak positive correlations of anthropometric parameters and 2 cytokines. Moreover, there was a trend slightly towards increased risk of T2DM with baseline IL- 6 and TNF- $\alpha$ levels in the highest tertile, while several studies have seen the elevated level of both cytokines as the risk of developing T2DM after adjusting for confounders [12, 40-43]. Nevertheless, there were unclear findings that the strength of relationship between 
IL-6 and T2DM in multivariate analysis was dramatically attenuated [9], and a nonstatistically significant result was found between TNF- $\alpha$ and T2DM risk [12]. Compared with CRP, IL- 6 and TNF- $\alpha$ showed weaker and more inconsistent associations with T2DM development. Both of them probably have greater variability and no obvious cut point.

Interestingly, we performed further analysis which indicated the combined effects of the three inflammatory markers on the 2-year risk of developing T2DM. A combined analysis obviously showed a significant interaction between baseline CRP and IL-6. The synergistic interaction between high levels of baseline CRP and IL-6 had 5.1 times elevated risk of developing T2DM, which was a 110 percent increase compared with the high levels of CRP alone. Study subjects with combined high levels of CRP and TNF- $\alpha$ were found to have an increased risk of T2DM; however, this effect was not strong enough to be determined as synergism. Our findings also demonstrated an effect of combined consideration between IL- 6 and TNF- $\alpha$. This seemed to be an antagonistic interaction in the condition of high IL- 6 and TNF- $\alpha$ levels. Likewise, interaction analysis of the cytokines in the EPICPotsdam study revealed that a combined elevation of IL-6 and TNF- $\alpha$ did not show a trend towards increased risk of developing T2DM [8]. These results suggested that the combination pattern of inflammatory cytokines may be related to the pathogenesis of T2DM. Our findings corresponded to the knowledge that inflammatory responses rely on several cytokines rather than on a single cytokine alone, and cytokines systematically operate in the form of a complex signaling network [35]. The influence of cytokines on insulin resistance may be considered with the combined elevation of various cytokines. Apart from IL- 6 and TNF- $\alpha$, other potential inflammatory cytokines, such as IL- $1 \beta$ and IL-8, have previously showed the effect of desensitization on insulin signaling via I $\kappa$ B kinase $\beta$ [44] and may be involved with poor glycemic control through activation of the Toll-like receptor 2 (TLR2) on B cells $[45,46]$.

In addition to cytokine levels at a single time, two consecutive examinations at the years 2013 and 2014 enable us to evaluate the relationship of IL- 6 and TNF- $\alpha$ changes with the risk of T2DM. The consistent 1-year high of IL- 6 and TNF- $\alpha$ levels was significantly associated with T2DM, although the risk attenuated after the adjustment for confounders. Moreover, a remarkable relationship was found between the risk of developing T2DM and increments of TNF- $\alpha$ level within 1 year. This result implies that insulin resistance probably occurs once TNF- $\alpha$ levels are initially risen at baseline and continuously elevated thereafter and may underline early detection of chronic low-grade inflammation levels in order to reduce the risk of developing T2DM. A longitudinal study with a longer follow-up period, more frequent examination, and larger sample size would be useful to clarify the effects of variation in inflammatory marker levels on T2DM progression.

\section{Conclusions}

Our 2-year retrospective analysis revealed that high baseline CRP levels were related with increased T2DM risk, and the impact was substantially greater when combined with other high inflammatory marker levels, particularly with IL-6. This study also showed strong relationship of 1-year TNF- $\alpha$ changes with T2DM progression. Thus, CRP and IL- 6 could be an effective combination for identifying as a T2DM risk factor. A one-year TNF- $\alpha$ variation may initiate T2DM and play a role as a potential predictive marker for T2DM. These results substantially support the role of inflammatory markers in the pathogenesis of T2DM and emphasize that the identification of biomarkers for predicting T2DM will be beneficial to develop effective diabetes prevention and an early detection program. The limitation of this study should be addressed. First, we cannot control the assessment of independent or dependent variables, because a retrospective design must rely on the accuracy of collected data from others. Second, a retrospective study may lead to selection bias and information bias. Third, the present study is a short-term retrospective cohort design; while this enabled it to investigate the associations for a short period, it may not provide reasonable information on longitudinal relationships of this chronic disease. Fourth, our study was performed with small sample sizes. Therefore, a future long-term cohort study with larger sample sizes is needed to clarify the predictive ability of these inflammatory markers on the risk of developing T2DM in rural Thais.

\section{Data Availability}

The data used to support the findings of this study are available from the corresponding author upon request.

\section{Conflicts of Interest}

The authors declare that there is no conflict of interest regarding the publication of this paper.

\section{Acknowledgments}

The authors particularly thank all the staff at Nong Waeng Health Promoting Hospital for assisting in collecting the data and samples, and we appreciate the collaboration of all participants in Sung Noen District, Nakhon Ratchasima Province, Thailand. This study was supported by the Faculty of Tropical Medicine, Mahidol University. The findings are part of a $\mathrm{PhD}$ program at Mahidol University.

\section{References}

[1] K. Bundhamcharoen, P. Odton, S. Phulkerd, and V. Tangcharoensathien, "Burden of disease in Thailand: changes in health gap between 1999 and 2004," BMC Public Health, vol. 11, no. 1, article 53, 2011.

[2] IDF, IDF Diabetes Atlas: Eighth Edition 2017, International Diabetes Federation, Brussels, Belgium, 2017.

[3] A. Thonghong, Annual epidemiological surveillance report, Bureau of Epidemiology, Ministry of Public Health, Bangkok, 2015.

[4] P. M. Ridker, "Clinical application of C-reactive protein for cardiovascular disease detection and prevention," Circulation, vol. 107, no. 3, pp. 363-369, 2003. 
[5] A. D. Pradhan, J. E. Manson, N. Rifai, J. E. Buring, and P. M. Ridker, "C-reactive protein, interleukin 6, and risk of developing type 2 diabetes mellitus," Journal of the American Medical Association, vol. 286, no. 3, pp. 327-334, 2001.

[6] A. Festa, R. D’Agostino, R. P. Tracy, and S. M. Haffner, "Elevated levels of acute-phase proteins and plasminogen activator inhibitor-1 predict the development of type 2 diabetes: the insulin resistance Atherosclerosis Study," Diabetes, vol. 51, no. 4, pp. 1131-1137, 2002.

[7] T. S. Han, N. Sattar, K. Williams, C. Gonzalez-Villalpando, M. E. J. Lean, and S. M. Haffner, "Prospective study of C-reactive protein in relation to the development of diabetes and metabolic syndrome in the Mexico City Diabetes Study," Diabetes Care, vol. 25, no. 11, pp. 2016-2021, 2002.

[8] J. Spranger, A. Kroke, M. Möhlig et al., "Inflammatory cytokines and the risk to develop type 2 diabetes: results of the prospective population-based European Prospective Investigation into Cancer and Nutrition (EPIC)-Potsdam Study," Diabetes, vol. 52, no. 3, pp. 812-817, 2003.

[9] F. B. Hu, J. B. Meigs, T. Y. Li, N. Rifai, and J. E. Manson, "Inflammatory markers and risk of developing type 2 diabetes in women," Diabetes, vol. 53, no. 3, pp. 693-700, 2004.

[10] H. Belfki, S. Ben Ali, S. Bougatef et al., "Association between $\mathrm{C}$-reactive protein and type 2 diabetes in a Tunisian population," Inflammation, vol. 35, no. 2, pp. 684-689, 2012.

[11] A. C. Dongway, A. S. Faggad, H. Y. Zaki, and B. E. Abdalla, "Creactive protein is associated with low-density lipoprotein cholesterol and obesity in type 2 diabetic Sudanese," Diabetes, Metabolic Syndrome and Obesity: Targets and Therapy, vol. 8, pp. 427-435, 2015.

[12] C. Phosat, P. Panprathip, N. Chumpathat et al., "Elevated Creactive protein, interleukin 6 , tumor necrosis factor alpha and glycemic load associated with type 2 diabetes mellitus in rural Thais: a cross-sectional study," BMC Endocrine Disorders, vol. 17, no. 1, p. 44, 2017.

[13] J. P. Wilding, "Obesity and nutritional factors in the pathogenesis of type 2 diabetes mellitus," in Textbook of Diabetes, J. C. Pickup, Ed., p. 21, Blackwell Science, Oxford, UK, 2003.

[14] M. A. Rupnick, D. Panigrahy, C.-Y. Zhang et al., "Adipose tissue mass can be regulated through the vasculature," Proceedings of the National Academy of Sciences of the United States of America, vol. 99, no. 16, pp. 10730-10735, 2002.

[15] P. Trayhurn and I. S. Wood, "Adipokines: inflammation and the pleiotropic role of white adipose tissue," British Journal of Nutrition, vol. 92, no. 3, pp. 347-355, 2004.

[16] G. S. Hotamisligil and E. Erbay, "Nutrient sensing and inflammation in metabolic diseases," Nature Reviews Immunology, vol. 8, no. 12, pp. 923-934, 2008.

[17] I. S. Wood, F. P. de Heredia, B. Wang, and P. Trayhurn, "Cellular hypoxia and adipose tissue dysfunction in obesity: symposium on 'Frontiers in Adipose Tissue Biology'," Proceedings of the Nutrition Society, vol. 68, no. 4, pp. 370-377, 2009.

[18] G. S. Hotamisligil, "Endoplasmic reticulum stress and the inflammatory basis of metabolic disease," Cell, vol. 140, no. 6, pp. 900-917, 2010.

[19] G. Murdolo and U. Smith, "The dysregulated adipose tissue: a connecting link between insulin resistance, type 2 diabetes mellitus and atherosclerosis," Nutrition, Metabolism and Cardiovascular Diseases, vol. 16, pp. S35-S38, 2006.
[20] M. F. Gregor and G. S. Hotamisligil, "Inflammatory mechanisms in obesity," Annual Review of Immunology, vol. 29, no. 1, pp. 415-445, 2011.

[21] G. R. Hajer, T. W. van Haeften, and F. L. J. Visseren, "Adipose tissue dysfunction in obesity, diabetes, and vascular diseases," European Heart Journal, vol. 29, no. 24, pp. 2959-2971, 2008.

[22] M. Banerjee and M. Saxena, "Genetic polymorphisms of cytokine genes in type 2 diabetes mellitus," World Journal of Diabetes, vol. 5, no. 4, pp. 493-504, 2014.

[23] G. S. Hotamisligil and B. M. Spiegelman, "Tumor necrosis factor $\alpha$ : a key component of the obesity-diabetes link," Diabetes, vol. 43, no. 11, pp. 1271-1278, 1994.

[24] G. S. Hotamisligil, P. Arner, J. F. Caro, R. L. Atkinson, and B. M. Spiegelman, "Increased adipose tissue expression of tumor necrosis factor-alpha in human obesity and insulin resistance," The Journal of Clinical Investigation, vol. 95, no. 5, pp. 2409-2415, 1995.

[25] A. E. Caballero, S. Arora, R. Saouaf et al., "Microvascular and macrovascular reactivity is reduced in subjects at risk for type 2 diabetes," Diabetes, vol. 48, no. 9, pp. 1856-1862, 1999.

[26] K. Popko, E. Gorska, A. Stelmaszczyk-Emmel et al., "Proinflammatory cytokines IL- 6 and TNF- $\alpha$ and the development of inflammation in obese subjects," European Journal of Medical Research, vol. 15, no. 2, article 120, 2010.

[27] T. L. Stanley, M. V. Zanni, S. Johnsen et al., "TNF- $\alpha$ antagonism with etanercept decreases glucose and increases the proportion of high molecular weight adiponectin in obese subjects with features of the metabolic syndrome," The Journal of Clinical Endocrinology \& Metabolism, vol. 96, no. 1, pp. E146-E150, 2011.

[28] J. J. Swaroop, D. Rajarajeswari, and J. N. Naidu, "Association of TNF- $\alpha$ with insulin resistance in type 2 diabetes mellitus," Indian Journal of Medical Research, vol. 135, no. 1, pp. 127130, 2012.

[29] S. Mirza, M. Hossain, C. Mathews et al., "Type 2-diabetes is associated with elevated levels of TNF-alpha, IL- 6 and adiponectin and low levels of leptin in a population of Mexican Americans: a cross-sectional study," Cytokine, vol. 57, no. 1, pp. 136-142, 2012.

[30] American Diabetes Association, "Classification and diagnosis of Diabetes," Diabetes Care, vol. 40, Supplement 1, pp. S11S24, 2016.

[31] Guidance for industry and FDA staff, "Review criteria for assessment of C-reactive protein (CRP), high sensitivity Creactive protein (hsCRP) and cardiac C-reactive protein (cCRP) assays," 2005, https://www.fda.gov/MedicalDevices/ ucm077167.htm.

[32] P. M. Ridker, N. Rifai, M. A. Pfeffer, F. Sacks, and E. Braunwald, "Long-term effects of pravastatin on plasma concentration of C-reactive protein," Circulation, vol. 100, no. 3, pp. 230235, 1999.

[33] I. S. Ockene, C. E. Matthews, N. Rifai, P. M. Ridker, G. Reed, and E. Stanek, "Variability and classification accuracy of serial high-sensitivity C-reactive protein measurements in healthy adults," Clinical Chemistry, vol. 47, no. 3, pp. 444-450, 2001.

[34] J. C. Pickup and M. A. Crook, "Is type II diabetes mellitus a disease of the innate immune system?," Diabetologia, vol. 41, no. 10, pp. 1241-1248, 1998.

[35] M. C. Calle and M. L. Fernandez, "Inflammation and type 2 diabetes," Diabetes \& Metabolism, vol. 38, no. 3, pp. 183191, 2012. 
[36] G. S. Hotamisligil, P. Peraldi, A. Budavari, R. Ellis, M. F. White, and B. M. Spiegelman, "IRS-1-mediated inhibition of insulin receptor tyrosine kinase activity in TNF- $\alpha$ - and obesityinduced insulin resistance," Science, vol. 271, no. 5249, pp. 665-670, 1996.

[37] V. Aguirre, E. D. Werner, J. Giraud, Y. H. Lee, S. E. Shoelson, and M. F. White, "Phosphorylation of Ser307 in insulin receptor substrate-1 blocks interactions with the insulin receptor and inhibits insulin action," Journal of Biological Chemistry., vol. 277, no. 2, pp. 1531-1537, 2002.

[38] S. E. Borst, "The role of TNF- $\alpha$ in insulin resistance," Endocrine, vol. 23, no. 2-3, pp. 177-182, 2004.

[39] S. Black, I. Kushner, and D. Samols, "C-reactive protein," Journal of Biological Chemistry, vol. 279, no. 47, pp. 48487-48490, 2004.

[40] B. B. Duncan, M. I. Schmidt, J. S. Pankow et al., "Low-grade systemic inflammation and the development of type 2 diabetes: the atherosclerosis risk in communities study," Diabetes, vol. 52, no. 7, pp. 1799-1805, 2003.

[41] P. Plomgaard, A. R. Nielsen, C. P. Fischer et al., "Associations between insulin resistance and TNF- $\alpha$ in plasma, skeletal muscle and adipose tissue in humans with and without type 2 diabetes," Diabetologia, vol. 50, no. 12, pp. 2562-2571, 2007.

[42] E.-S. Yeo, J.-Y. Hwang, J. E. Park, Y. J. Choi, K. B. Huh, and W. Y. Kim, "Tumor necrosis factor (TNF- $\alpha$ ) and C-reactive protein (CRP) are positively associated with the risk of chronic kidney disease in patients with type 2 diabetes," Yonsei Medical Journal, vol. 51, no. 4, pp. 519-525, 2010.

[43] G. Daniele, R. Guardado Mendoza, D. Winnier et al., "The inflammatory status score including IL-6, TNF- $\alpha$, osteopontin, fractalkine, MCP-1 and adiponectin underlies whole-body insulin resistance and hyperglycemia in type 2 diabetes mellitus," Acta Diabetologica, vol. 51, no. 1, pp. 123-131, 2014.

[44] S. E. Shoelson, J. Lee, and M. Yuan, "Inflammation and the $\mathrm{IKK} \beta / \mathrm{I} \kappa \mathrm{B} / \mathrm{NF}-\kappa \mathrm{B}$ axis in obesity- and diet-induced insulin resistance," International Journal of Obesity, vol. 27, no. S3, pp. S49-S52, 2003.

[45] M. R. Dasu, S. Devaraj, L. Zhao, D. H. Hwang, and I. Jialal, "High glucose induces toll-like receptor expression in human monocytes. Mechanism of Activation," Diabetes, vol. 57, no. 11, pp. 3090-3098, 2008.

[46] M. Jagannathan, M. McDonnell, Y. Liang et al., "Toll-like receptors regulate $\mathrm{B}$ cell cytokine production in patients with diabetes," Diabetologia, vol. 53, no. 7, pp. 1461-1471, 2010. 


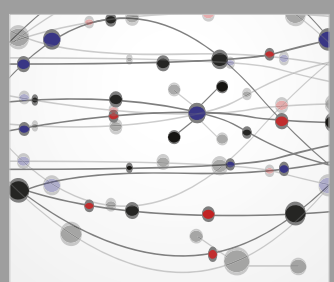

The Scientific World Journal
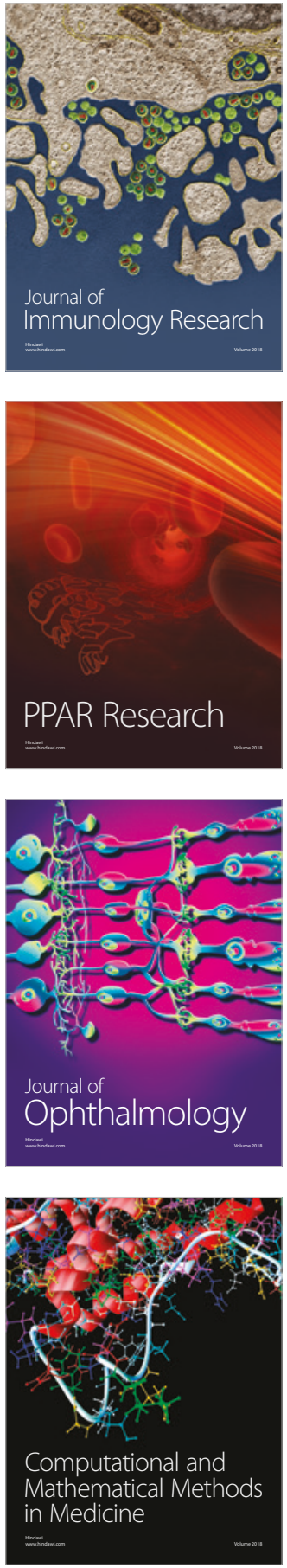

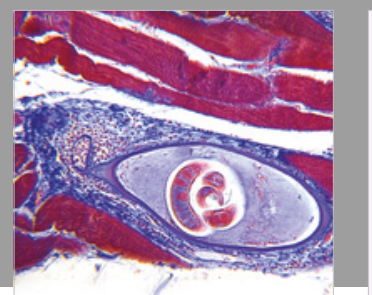

Gastroenterology Research and Practice

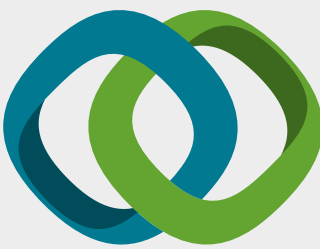

\section{Hindawi}

Submit your manuscripts at

www.hindawi.com
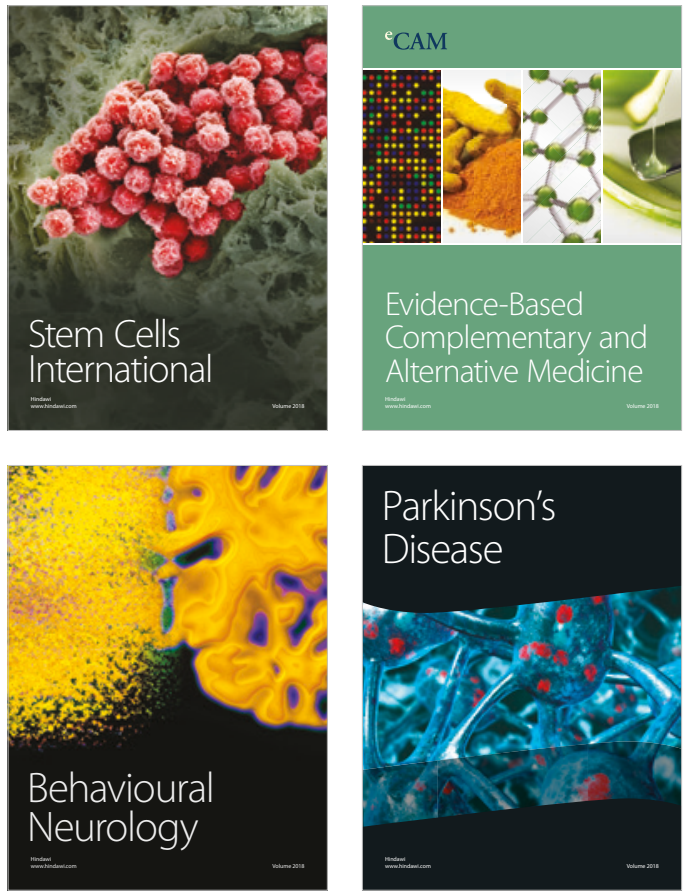

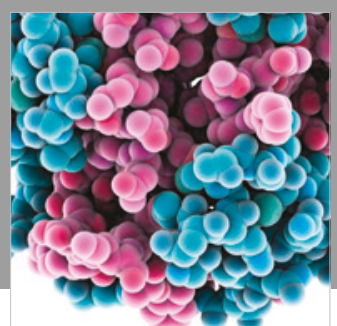

ournal of

Diabetes Research

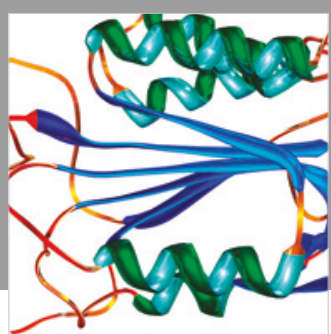

Disease Markers
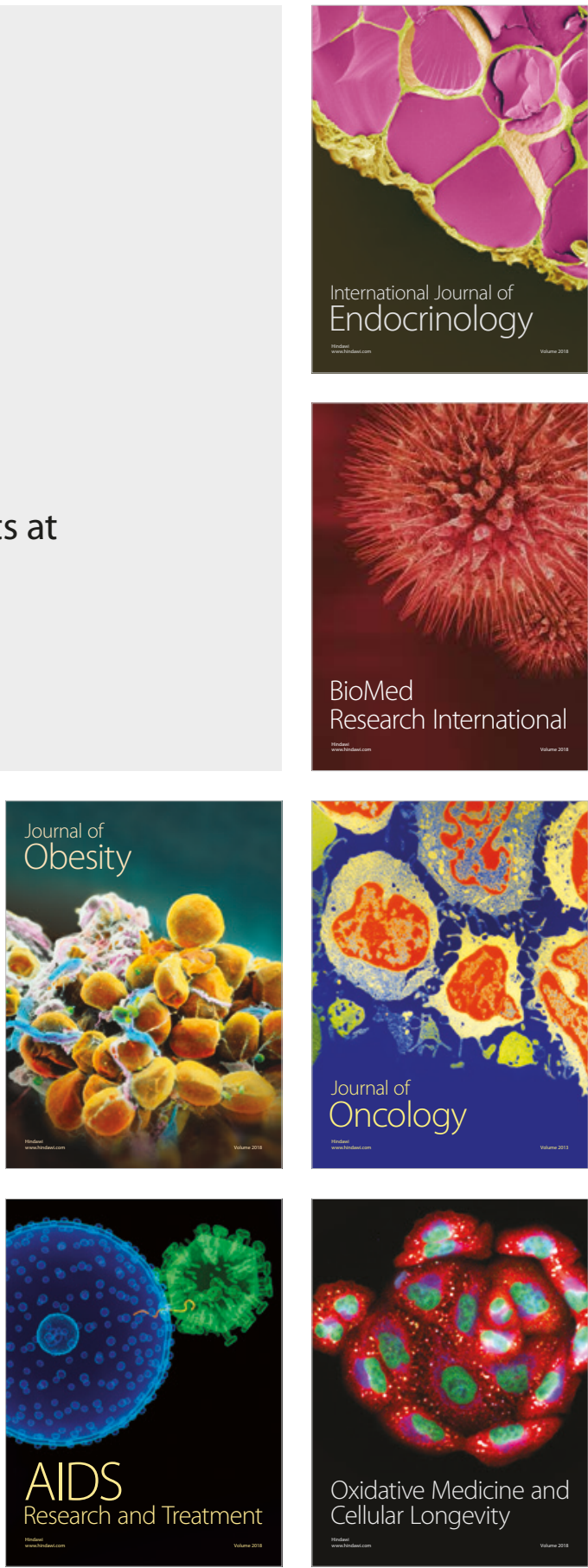